\title{
The COVID-19 Home Environment Literacy Practices (COVID19-HELP) Questionnaire
}

Carolyn J. King ${ }^{1}$, Ally M. Lee ${ }^{1}$, Jennifer $Z^{1} k^{1,2}$, Nivedita Ravi ${ }^{1}$, Nadine Gaab ${ }^{1,2,3}$

${ }^{1}$ Laboratories of Cognitive Neuroscience, Division of Developmental Medicine, Boston

Children's Hospital, Boston, Massachusetts, U.S.

${ }^{2}$ Harvard Medical School, Boston, Massachusetts, U.S.

${ }^{3}$ Harvard Graduate School of Education, Cambridge, Massachusetts, U.S.

Correspondence:

Nadine Gaab, PhD

Laboratories of Cognitive Neuroscience

Department of Medicine/Division of Developmental Medicine

Boston Children's Hospital

1 Autumn Street

Boston, MA 02215

nadine.gaab@childrens.harvard.edu 


\section{Introduction}

The COVID-19 Home Environment Literacy Practices (COVID19-HELP) Questionnaire was designed to examine the impact of the novel coronavirus (COVID-19; also called SARS-CoV-2) pandemic restrictions and school closures on home literacy environment and other enrichment practices such as remote school work, educational activities and musical exposure in the home. Caregivers with children between ages 0-11 are asked questions regarding their home literacy environment and practices with their children as well as other enrichments related to literacy, before and since COVID-19 affected their community. Respondents also indicate how their job and home situations have changed since the COVID-19 pandemic. Additionally, the questionnaire captures relevant demographic information, including family history of reading difficulties, socioeconomic status, race, and geographic location. To request access to our data dictionary, please visit http://gaablab.com/contact.

\section{Questionnaires}

Our questionnaire builds upon previous research related to home literacy environment and other enrichment activities. We adapted a Home Literacy Environment (HLE) questionnaire to evaluate style of reading (Reese, 1999; Roberts, 2005); frequency of formal and informal literacy activities (Bus, 1995; Evans, 2000; Purcell-Gates, 1996; Scarborough \& Dobrich, 1994); physical environment (Morrow, 1990; Neuman, 1997; Vukelich, 1994); and parents' literacy activities modeled at home (van Steensel, 2006). In addition to aspects of HLE, we also included information about enrichments related to literacy (e.g., music), socioeconomic (SES) factors, family history of reading difficulties, and current job and home situations related to the COVID-19 pandemic (CRISIS survey). Some questions were adapted from the Coronavirus Health Impact Survey (CRISIS), attribution license CC-BY-4.0 (https://creativecommons.org/licenses/by/4.0/), from the research teams of Kathleen Merikangas and Argyris Stringaris at the National Institute of Mental Health Intramural Research Program Mood Spectrum Collaboration, and those of Michael P. Milham at the Child Mind Institute and the NYS Nathan S. Kline Institute for Psychiatric Research. 


\section{References}

Bus, A. G., van Ijzendoorn, M.H., \& Pellegrini, A.D. (1995). Joint book reading makes for success in learning to read: A meta-analysis of intergenerational transmission of literacy. Review of Educational Research, 65(1), 1-22.

Evans, M. A., Shaw, D., \& Bell, M. (2000). Home literacy activities and their influence on early literacy skills. Can J Exp Psychol, 54(2), 65-75. Retrieved from http://www.ncbi.nlm.nih.gov/pubmed/10881391

Morrow, L. (1990). Preparing the classroom environment to promote literacy during play. Early Childhood Research Quarterly, 5, 537-544.

Neuman, S., \& Roskos, K. (1997). Literacy knowledge in practice: Contexts of participation for young writers and readers. Reading Research Quarterly, 32, 10-32.

Purcell-Gates, V. (1996). Stories, coupons, and the "TV guide": Relationships between home literacy experiences and emergency literacy knowledge. Reading Research Quarterly, 31(4), 406-428.

Reese, E., \& Cox, A. (1999). Quality of adult book reading affects children's emergent literacy. Dev Psychol, 35(1), 20-28. Retrieved from http://www.ncbi.nlm.nih.gov/pubmed/9923461

Roberts, J., Jurgens, J., \& Burchinal, M. (2005). The role of home literacy practices in preschool children's language and emergent literacy skills. J Speech Lang Hear Res, 48(2), 345359. Retrieved from http://www.ncbi.nlm.nih.gov/entrez/query.fcgi?cmd=Retrieve \&db=PubMed\&dopt=Citati on\&lisl_uids $=15989397$

Scarborough, H. S., Dobrich, W., \& Hager, M. (1991). Preschool literacy experience and later reading achievement. J Learn Disabil, 24(8), 508-511. Retrieved from http://www.ncbi.nlm.nih.gov/entrez/query.fcgi?cmd=Retrieve \&db=PubMed\&dopt=Citati on\&list uids $=1940609$

van Steensel, R. (2006). Relations between socio-cultural factors, the home literacy environment and children's literacy development in the first years of primary education. Journal of Research in Reading, 29(4), 367-382. https://doi.org/10.1111/j.1467-9817.2006.00301.x

Vukelich, C. (1994). Effects of play interventions on young children's reading of environmental print. Early Childhood Research Quarterly, 9, 153-170. 


\section{Background}




\section{Background Questions}

We ask that you complete a series of six surveys of varying lengths. Once you click "Submit" on one survey, the next survey will appear. Completion of all surveys will take approximately 30 minutes of your time.If you are on a smart phone, please change your screen orientation to landscape (if possible).In this study, the novel coronavirus of 2019/2020 is referred to as COVID-19.

Today's date

How many people lived in your home (including yourself) before COVID-19 affected your community?
$\bigcirc 2$
$\bigcirc 3$
$\bigcirc 4$
$\bigcirc 5$
$\bigcirc 6$
$\bigcirc 7$
O 8 or more

How many people currently live in your home (including yourself) since COVID-19 affected your community?
$\bigcirc 2$
$\bigcirc 3$
$\bigcirc 4$
○ 5
$\bigcirc 6$
$\bigcirc 7$
$\bigcirc 8$ or more

How many children in your home are between ages 0-11?
$\bigcirc 1$
$\bigcirc 2$
$\bigcirc 3$
$\bigcirc 4$
5 or more

Please indicate the first initial of your oldest (or only) child between ages 0-11 (e.g., Charlotte =C).

We will use this initial throughout the survey when

referring to this child, and it will be displayed in blue.

note: If you have multiple children with the same

first initial, please indicate the first two letters

of their first name (e.g., Jane = Ja; John = Jo).

How old is [initial_child1]?

$\bigcirc 0$ years old

2 years old

$\bigcirc 4$ years old

6 years old

8 years old

$\bigcirc 10$ years old

$\bigcirc 1$ year old

3 years old

5 years old

7 years old

9 years old

11 years old

Has [initial_child1] started formal reading instruction (e.g., kindergarten in the U.S. or first grade in many European countries)?

Y Yes

No 
How many years of formal education will

[initial_child1] have completed in 2020?
1 year (finished preschool)

2 years (finished kindergarten)

3 years (finished first grade)

4 years (finished second grade)

5 years (finished third grade)

6 years (finished fourth grade)

7 years (finished fifth grade)

8 years (finished sixth grade)

9 years (finished seventh grade)

10 years or greater (finished finished eighth grade or greater)

Please indicate the first initial of your second oldest child between ages 0-11 (e.g., Charlotte $=$ C). We will use this initial throughout the survey when referring to this child, and it will be displayed in pink.

note: If you have multiple children with the same first initial, please indicate the first two letters of their first name (e.g., Jane = Ja; John $=$ Jo).

How old is [initial_child2]?

0 years old

2 years old

$\bigcirc$ years old

6 years old

8 years old

10 years old

1 year old

3 years old

5 years old

7 years old

$\bigcirc 9$ years old

$\bigcirc 11$ years old

Has [initial_child2] started formal reading instruction (e.g., kindergarten in the U.S. or first grade in many European countries)?

OYes

No
How many years of formal education will [initial_child2] have completed in 2020?

How many years of formal education will

[initial_child2] have completed in 2020?
0 years

1 year (finished preschool)
1 year (finished preschool)

$\bigcirc 2$ years (finished kindergarten)

$\bigcirc 3$ years (finished first grade)

$\bigcirc 4$ years (finished second grade)

5 years (finished third grade)

6 years (finished fourth grade)

$\bigcirc 7$ years (finished fifth grade)

$\bigcirc 8$ years (finished sixth grade)

9 years (finished seventh grade) 10 years or greater (finished finished eighth
grade or greater)

Please indicate the first initial of your third oldest child between ages 0-11 (e.g., Charlotte = C). We will use this initial throughout the survey when referring to this child, and it will be displayed in green.

note: If you have multiple children with the same first initial, please indicate the first two letters of their first name (e.g., Jane = Ja; John = Jo). 
How old is [initial_child3]?
$\bigcirc 0$ years old
2 years old
4 years old
6 years old
8 years old
10 years old
1 year old
3 years old
5 years old
7 years old
$\bigcirc$ years old
11 years old

Has [initial_child3] started formal reading instruction (e.g., kindergarten in the U.S. or first grade in many European countries)?

$\bigcirc$ Yes

No

How many years of formal education will [initial_child3] have completed in 2020?

00 years

1 year (finished preschool)

How many years of formal education will

[initial_child3] have completed in 2020?

1 year (finished preschool)

2 years (finished kindergarten)

3 years (finished first grade)

$\bigcirc 4$ years (finished second grade)

$\bigcirc 5$ years (finished third grade)

$\bigcirc 6$ years (finished fourth grade)

$\bigcirc 7$ years (finished fifth grade)

$\bigcirc 8$ years (finished sixth grade)

$\bigcirc 9$ years (finished seventh grade)

$\bigcirc 10$ years or greater (finished finished eighth grade or greater)

Please indicate the first initial of your fourth oldest child between ages 0-11 (e.g., Charlotte $=$ C). We will use this initial throughout the survey when referring to this child, and it will be displayed in purple.

note: If you have multiple children with the same first initial, please indicate the first two letters of their first name (e.g., Jane = Ja; John = Jo).

How old is [initial_child4]?

$\bigcirc 0$ years old

2 years old

4 years old

6 years old

8 years old

$\bigcirc 10$ years old
1 year old

3 years old

5 years old

7 years old

9 years old

$\bigcirc 11$ years old

Has [initial_child4] started formal reading instruction (e.g., kindergarten in the U.S. or first grade in many European countries)?

$\bigcirc$ Yes
No 
How many years of formal education will [initial_child4] have completed in 2020?
1 year (finished preschool)

2 years (finished kindergarten)

3 years (finished first grade)

4 years (finished second grade)

5 years (finished third grade)

6 years (finished fourth grade)

7 years (finished fifth grade)

8 years (finished sixth grade)

9 years (finished seventh grade)

10 years or greater (finished finished eighth grade or greater)

Please indicate the first initial of your fifth oldest child between ages 0-11 (e.g., Charlotte $=$ C). We will use this initial throughout the survey when referring to this child, and it will be displayed in red.

note: If you have multiple children with the same first initial, please indicate the first two letters of their first name (e.g., Jane = Ja; John $=$ Jo).

How old is [initial_child5]?

0 years old

2 years old

4 years old

6 years old

8 years old

10 years old
1 year old

3 years old

5 years old

7 years old

$\bigcirc 9$ years old

$\bigcirc 11$ years old

Has [initial_child5] started formal reading instruction (e.g., kindergarten in the U.S. or first grade in many European countries)?

Yes

No
How many years of formal education will [initial_child5] have completed in 2020?
0 years

1 year (finished preschool)
How many years of formal education will [initial_child5] have completed in 2020?
1 year (finished preschool)

2 years (finished kindergarten)

3 years (finished first grade)

4 years (finished second grade)

5 years (finished third grade)

6 years (finished fourth grade)

7 years (finished fifth grade)

8 years (finished sixth grade)

9 years (finished seventh grade)

10 years or greater (finished finished eighth grade or greater) 
Please indicate your relationship to your child(ren). You may check all that apply.
Biological mother
Biological father
Step-mother
Step-father
Grandmother
Grandfather
Adoptive mother
Adoptive father
Foster mother
Foster father
Aunt
Uncle
Guardian
Other

Please specify:

How many adults in your household (including child care professionals working in your home) provided daily care to your child(ren) before COVID-19 affected your community? (e.g., help with daily activities, cooking for child(ren), child rearing).

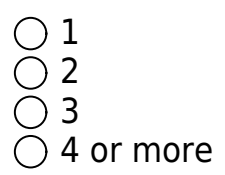

Please include yourself in this number.

Please provide the initials of the other adult(s) (not including you) who provided care to your child(ren) before COVID-19 affected your community.

Please indicate the first initial of the second adult who provided care to your child(ren) before COVID-19

affected your community (e.g., Charlotte $=\mathrm{C}$ ).note:

If there are multiple adults who provided care to your child(ren) with the same first initial, please indicate the first two letters of their first name (e.g., Jane = Ja; John = Jo).

Please indicate the first initial of the third adult who provided care to your child(ren) before COVID-19 affected your community (e.g., Charlotte = C). note: If there are multiple adults who provided care to your child(ren) with the same first initial, please indicate the first two letters of their first name (e.g., Jane = Ja; John = Jo).

Please indicate the first initial of the fourth adult who provided care to your child(ren) before COVID-19 affected your community (e.g., Charlotte = C). note: If there are multiple adults who provided care to your child(ren) with the same first initial, please indicate the first two letters of their first name (e.g., Jane = Ja; John = Jo). 
How many adults in your household (including child care professionals working in your home) provided daily care to your child(ren) since COVID-19 affected your community? (e.g., help with daily activities, cooking for child(ren), child rearing). Please include yourself in this number.

Please provide the initials of the other adult(s) (not including you) who provide(s) care to your child(ren) since COVID-19 affected your community.

Please indicate the first initial of the second adult who provides care to your child(ren) since COVID-19 affected your community (e.g., Charlotte $=$ C). note: If there are multiple adults who provide care to your child(ren) with the same first initial, please indicate the first two letters of their first name (e.g., Jane = Ja; John = Jo).

Please indicate the first initial of the third adult who provides care to your child(ren) since COVID-19 affected your community (e.g., Charlotte $=C$ ). note: If there are multiple adults who provide care to your child(ren) with the same first initial, please indicate the first two letters of their first name (e.g., Jane = Ja; John = Jo).

Please indicate the first initial of the fourth adult who provides care to your child(ren) since COVID-19 affected your community (e.g., Charlotte $=\mathrm{C}$ ). note: If there are multiple adults who provide care to your child(ren) with the same first initial, please indicate the first two letters of their first name (e.g., Jane = Ja; John = Jo).

What was your job situation before COVID-19 affected your community? Please check all that apply.
$\bigcirc 1$
$\bigcirc 2$
$\bigcirc 3$
4 or more 
What is your job situation now, since COVID-19

affected your community? Please check all that apply.
Work outside the home full-time ( $>35 \mathrm{hrs} /$ week)

Work from home full-time ( $>35 \mathrm{hrs} /$ week)

Work partly from home full-time (>35 hrs/week)

$\square$ Work outside the home part-time (<35 hrs/week)

$\square$ Work from home part-time (< $35 \mathrm{hrs} /$ week)

Student

On temporary leave (e.g., furlough, maternity leave)

$\square$ Keep house or raise children full-time

$\square$ Unemployed

Retired

On disability (disability benefits)

Prefer not to answer
What was the job situation of

[bg_caregivers_name_b4_pg2] before COVID-19 affected your community? Please check all that apply.
Worked outside the home full-time (>35 hrs/week) Worked from home full-time ( $>35 \mathrm{hrs} /$ week)

Worked partly from home full-time (>35 hrs/week)

Worked outside the home part-time ( $<35 \mathrm{hrs} /$ week)

$\square$ Worked from home part-time (<35 hrs/week)

Worked in my home full-time as a child care provider

Worked in my home part-time as a child care provider

Student

$\square$ On temporary leave (e.g., furlough, maternity leave)

$\square$ Kept house or raised children full-time

$\square$ Unemployed

$\square$ Retired

On disability (disability benefits)

Prefer not to answer
What is the job situation of

[bg_caregivers_name_since_pg2], since COVID-19

affected your community? Please check all that apply.
Work outside the home full-time (>35 hrs/week)

Work from home full-time ( $>35 \mathrm{hrs} /$ week)

$\square$ Work partly from home full-time (>35 hrs/week)

$\square$ Work outside the home part-time (<35 hrs/week)

Work from home part-time ( $<35 \mathrm{hrs} /$ week)

$\square$ Work in my home full-time as a child care provider

Work in my home part-time as a child care provider

Student

$\square$ On temporary leave (e.g., furlough, maternity leave)

Keep house or raise children full-time

$\square$ Unemployed

Retired

On disability (disability benefits)

Prefer not to answer 
What was the job situation of

[bg caregivers name b4 pg3] before COVID-19 affected your community? Please check all that apply.
Worked outside the home full-time (>35 hrs/week) Worked from home full-time (>35 hrs/week)

$\square$ Worked partly from home full-time (>35 hrs/week)

$\square$ Worked outside the home part-time (<35 hrs/week)

$\square$ Worked from home part-time (< 35 hrs/week)

Worked in my home full-time as a child care provider

Worked in my home part-time as a child care provider

Student

$\square$ On temporary leave (e.g., furlough, maternity leave)

Kept house or raised children full-time

$\square$ Unemployed

Retired

On disability (disability benefits)

Prefer not to answer
What is the job situation of

[bg_caregivers_name_since_pg3] now, since COVID-19

affected your community? Please check all that apply.
Work outside the home full-time (>35 hrs/week)

Work from home full-time (>35 hrs/week)

$\square$ Work partly from home full-time (>35 hrs/week)

$\square$ Work outside the home part-time (<35 hrs/week)

Work from home part-time (<35 hrs/week)

$\square$ Work in my home full-time as a child care provider

$\square$ Work in my home part-time as a child care provider

Student

$\square$ On temporary leave (e.g., furlough, maternity leave)

$\square$ Keep house or raise children full-time

Unemployed

Retired

On disability (disability benefits)

Prefer not to answer
What was the job situation of

[bg_caregivers_name_b4_pg4] before COVID-19 affected

your community? Please check all that apply.
Worked outside the home full-time (>35 hrs/week)

Worked from home full-time (>35 hrs/week)

Worked partly from home full-time (>35 hrs/week)

Worked outside the home part-time ( $<35 \mathrm{hrs} /$ week)

Worked from home part-time (<35 hrs/week)

Worked in my home full-time as a child care provider

$\square$ Worked in my home part-time as a child care provider

$\square$ Student

$\square$ On temporary leave (e.g., furlough, maternity leave)

$\square$ Kept house or raised children full-time

$\square$ Unemployed

Retired

On disability (disability benefits)

Prefer not to answer 
What is the job situation of

[bg_caregivers_name_since_pg4] now, since COVID-19 affected your community? Please check all that apply.
Work outside the home full-time ( $>35 \mathrm{hrs} /$ week)

Work from home full-time (>35 hrs/week)

$\square$ Work partly from home full-time ( $>35 \mathrm{hrs} /$ week)

Work outside the home part-time (<35 hrs/week)

Work from home part-time ( $<35$ hrs/week)

Work in my home full-time as a child care provider

Work in my home part-time as a child care provider Student

On temporary leave (e.g., furlough, maternity leave)

$\square$ Keep house or raise children full-time

$\square$ Unemployed

Retired

On disability (disability benefits)

Prefer not to answer
Which of the following restrictions have been placed on you and your family as a result of the COVID-19 outbreak? Please check all that apply.
No restrictions currently

Self-imposed quarantine or isolation

$\square$ Stay-at-home order or advisory by local government and/or employer (i.e., walks and errands with social distancing permitted)

$\square$ Shelter-in-place order by local government (i.e., only permitted outdoors for essential purposes)

Other

Please specify:

How stressful has staying at home been for you and your family?

Not at all

Slightly

Moderately

Very

Extremely

\begin{tabular}{ll}
\hline Have schools closed in your area? & $\bigcirc$ Yes \\
& $\bigcirc$ No
\end{tabular}

As far as you know, approximately when is your child(ren)'s school opening up again?
Start date in this school year

Closed until the end of this school year

Closed into the next academic school year

Unsure

Do you have access to a smartphone at home?

Yes

No

Before COVID-19 affected your community, how much time did you spend on your phone per day? (e.g., reading texts, emails, or articles; making phone calls; scrolling social media; playing games)
Less than $1 \mathrm{hr}$

$1 \mathrm{hr}-1 \mathrm{hr} 59 \mathrm{~min}$

2 hrs - 2 hrs 59 min

$3 \mathrm{hrs}-3 \mathrm{hrs} 59 \mathrm{~min}$

$4 \mathrm{hrs}-4 \mathrm{hrs} 59 \mathrm{~min}$

5 hrs - 5 hrs 59 min

6 hrs - 6 hrs 59 min

More than $7 \mathrm{hrs}$

Not applicable 
Since COVID-19 affected your community, how much time do you spend on your phone per day? (e.g., reading texts, emails, or articles; making phone calls; scrolling social media; playing games)
Less than $1 \mathrm{hr}$

$1 \mathrm{hr}-1 \mathrm{hr} 59 \mathrm{~min}$

2 hrs - 2 hrs 59 min

$3 \mathrm{hrs}-3 \mathrm{hrs} 59 \mathrm{~min}$

4 hrs - 4 hrs 59 min

$5 \mathrm{hrs}-5 \mathrm{hrs} 59 \mathrm{~min}$

6 hrs - 6 hrs 59 min

More than 7 hrs

Not applicable

$\begin{array}{ll}\text { Do you have access to a tablet at home (e.g., iPad, } & \bigcirc \text { Yes } \\ \text { Amazon Fire, not an e-reader like a Kindle)? } & \bigcirc \text { No }\end{array}$

Do you have access to a computer at home (e.g., laptop, desktop, chromebook)?

Yes

No

Did you have access to the internet (e.g., Wi-Fi,

DSL, not including cellular) at home before COVID-19

affected your community?

Yes

No

Do you have access to the internet (e.g., Wi-Fi, DSL, not including cellular) at home now, since COVID-19 affected your community?

On a typical day, how much time did your child(ren) spend outside before COVID-19 affected your community?
○ 1 - $30 \mathrm{~min}$

$31-60 \mathrm{~min}(1 \mathrm{hr})$

61 - $90 \mathrm{~min}$ (1.5 hrs)

91 - $120 \mathrm{~min}$ (2 hrs)

$121-150 \mathrm{~min}$ ( $2.5 \mathrm{hrs}$ )

$151-180 \mathrm{~min}$ (3 hrs)

Over 3 hrs

Not applicable
On a typical day, how much time does your child(ren) spend outside since COVID-19 affected your community?
$1-30 \mathrm{~min}$

$31-60 \mathrm{~min}(1 \mathrm{hr})$

$\bigcirc 61-90 \mathrm{~min}$ (1.5 hrs)

91 - $120 \mathrm{~min}$ (2 hrs)

$121-150 \mathrm{~min}$ (2.5 hrs)

151 - 180 min (3 hrs)

Over 3 hrs

Not applicable

How many languages, including English, are spoken at home?

$\bigcirc 1$
$\bigcirc 2$
$\bigcirc 3$
$\bigcirc$
$\bigcirc$
$\bigcirc$
6 or more

Please indicate the name of the second language (after English) spoken at home.

Please indicate the name of the third language spoken at home. 
Please indicate the name of the fourth language spoken at home.

Please indicate the name of the fifth language spoken at home.

Please indicate the name of the sixth language spoken at home.

Before COVID-19 affected your community, what percentage of speaking was done in each language?

Percentage in English:

Percentage in [bg_language2]:

Percentage in [bg_language3]:

Percentage in [bg_language4]:

Percentage in [bg_language5]:

Percentage in [bg_language6]:

\section{Since COVID-19 affected your community, what percentage of speaking is done in each} language?

Percentage in English:

Percentage in [bg_language2]:

Percentage in [bg_language3]:

Percentage in [bg_language4]:

Percentage in [bg_language5]:

Percentage in [bg_language6]: 
Before COVID-19 affected your community, what percentage of reading was done in each language?

Percentage in English:

Percentage in [bg_language2]:

Percentage in [bg_language3]:

Percentage in [bg_language4]:

Percentage in [bg_language5]:

Percentage in [bg_language6]:

Since COVID-19 affected your community, what percentage of reading is done in each language?

Percentage in English:

Percentage in [bg_language2]:

Percentage in [bg_language3]:

Percentage in [bg_language4]:

Percentage in [bg_language5]:

Percentage in [bg_language6]:

Please review your responses for completion and accuracy. You cannot go back once you've hit "Submit".

Survey completion: 1/6 


\section{Family History}




\section{Family History}

Do any immediate family members have a history of the following difficulties? This applies only to your child's biological/genetic relatives. $\square$ Reading difficulties (e.g., difficulty reading and comprehending written text)

$\square$ Spelling difficulties (e.g., difficulty splitting words into letters; difficulty with focusing on spelling)

$\square$ Math (numeracy, arithmetic) difficulties (e.g., difficulty with simple math concepts like "less" or "more;" difficulty putting things in order; difficulty understanding distance or time)

$\square$ Speech difficulties (e.g., stuttering; difficulty making mouth and tongue to speak)

$\square$ Language difficulties (e.g., difficult to follow along in conversation; limited vocabulary; unable to follow verbal instructions)
Does [initial_child1] have difficulties with vision or hearing?
Yes
No

\begin{tabular}{ll}
\hline Have these difficulties been corrected? (e.g., & $\bigcirc$ Yes \\
glasses, hearing aids) & $\bigcirc$ No
\end{tabular}
To your knowledge, is [initial_child1]'s reading
development progressing normally along with his/her
Yes
same-aged peers?
No
Not Applicable

Please specify:
Does [initial_child2] have difficulties with vision or hearing?
Yes
No
Have these difficulties been corrected? (e.g.,
glasses, hearing aids)
Yes
No

To your knowledge, is [initial_child2]'s reading development progressing normally along with his/her same-aged peers?
$\bigcirc$ Yes
No
$\bigcirc$ Not Applicable

Please specify:
Does [initial child3] have difficulties with vision or hearing?
Y Yes
No
Have these difficulties been corrected? (e.g., glasses, hearing aids)
Yes
No

To your knowledge, is [initial_child3]'s reading development progressing normally along with his/her same-aged peers?

Yes
No
Not Applicable


Please specify:

Does [initial_child4] have difficulties with vision or hearing?

OYes

No

Have these difficulties been corrected? (e.g., glasses, hearing aids)

Yes

No

To your knowledge, is [initial_child4]'s reading

Yes

development progressing normally along with his/her

No

same-aged peers?

Not Applicable

Please specify:

Does [initial_child5] have difficulties with vision or hearing?

$\bigcirc$ Yes

No

Have these difficulties been corrected? (e.g., glasses, hearing aids)

$\bigcirc$ Yes

No

To your knowledge, is [initial_child5]'s reading development progressing normally along with his/her same-aged peers?

Yes

No

Not Applicable

Please specify:

Please review your responses for completion and accuracy. You cannot go back once you've hit "Submit".

Survey completion: $2 / 6$ 


\section{Reading History}




\section{Reading History}

These questions pertain to your child(ren)'s exposure to literacy and other enrichments related to literacy at home. Some of these questions may not be relevant to your child(ren)'s age(s). We do not expect you to have done all of these activities with your child(ren). Please answer, to your best ability, based on the timeframe cited in each question - either right before COVID-19 affected your community, or since COVID-19 affected your community.

At what age was [initial_child1] first read to?
Prenatal
0 - 4 months
5 - 9 months
10 - 14 months
15 - 19 months
20 - 24 months
Older than 24 months
Unknown/unsure

How often was [initial_child1] read to before COVID-19 affected your community?
Never
1 - 3 times per month
1 - 2 times per week
3 - 4 times per week
Almost every day
1 - 2 times per day
3 or more times a day

How often is [initial_child1] read to since COVID-19

affected your community?

Never

1 - 3 times per month

1 - 2 times per week

3 - 4 times per week

Almost every day

1 - 2 times per day

3 or more times a day

\section{How often did the following occur before COVID-19 affected your community?}

\begin{tabular}{|c|c|c|c|c|c|c|}
\hline Never & $\begin{array}{l}1 \text { - } 3 \text { times } \\
\text { per month }\end{array}$ & $\begin{array}{l}1 \text { - } 2 \text { times } \\
\text { per week }\end{array}$ & $\begin{array}{l}3 \text { - } 4 \text { times } \\
\text { per week }\end{array}$ & $\begin{array}{c}\text { Almost } \\
\text { every day }\end{array}$ & $\begin{array}{c}1 \text { - } 2 \text { times } \\
\text { per day }\end{array}$ & $\begin{array}{l}3 \text { or more } \\
\text { times a day }\end{array}$ \\
\hline
\end{tabular}

You read to [initial_child1] (e.g., you read aloud and your child listened)

You read with [initial_child1] (e.g., child read the text with you)

[initial_child1] read

independently (e.g., child read a

book without help from you)

$\begin{array}{ccc}0 & 0 & \bigcirc \\ 0 & 0 & \bigcirc \\ 0 & 0 & \bigcirc\end{array}$




\section{How often has the following occurred since COVID-19 affected your community?}

$\begin{array}{cccccc}\text { Never } & 1-3 \text { times } & 1-2 \text { times } & 3-4 \text { times } & \text { Almost } & 1-2 \text { times } \\ & \text { per month } & \text { per week } & \text { per week } & \text { every day } & \text { per day times a day }\end{array}$

You read to [initial_child1] (e.g., you read aloud and your child

$\mathrm{O}$

$\bigcirc$

listens)

You read with [initial_child1] (e.g., child reads the text with you)

[initial_child1] reads independently (e.g., child reads a book without help from you)

On a typical day, how much was [initial_child1] read to before COVID-19 affected your community?
$1-30 \mathrm{~min}$

$31-60 \mathrm{~min}(1 \mathrm{hr})$

$61-90$ min (1.5 hrs)

91 - $120 \mathrm{~min}$ (2 hrs)

121 - $150 \mathrm{~min}$ (2.5 hrs)

$151-180 \mathrm{~min}$ (3 hrs)

Over 3 hrs

Not applicable
On a typical day, how much is [initial child1] read to since COVID-19 affected your community?
$1-30 \mathrm{~min}$

$31-60 \mathrm{~min}(1 \mathrm{hr})$

61 - 90 min (1.5 hrs)

91 - $120 \mathrm{~min}$ (2 hrs)

$121-150 \mathrm{~min}$ ( $2.5 \mathrm{hrs})$

151 - 180 min (3 hrs)

$\bigcirc$ Over 3 hrs

Not applicable

At what age was [initial_child2] first read to?

Prenatal

0 - 4 months

5 - 9 months

10 - 14 months

15 - 19 months

20 - 24 months

Older than 24 months

$\bigcirc$ Unknown/unsure
How often was [initial child2] read to before COVID-19 affected your community?
Never

1 - 3 times per month

1 - 2 times per week

3 - 4 times per week

Almost every day

1 - 2 times per day

3 or more times a day 
How often is [initial_child2] read to since COVID-19 affected your community?

Never

1 - 3 times per month

1 - 2 times per week

3 - 4 times per week

Almost every day

1 - 2 times per day

3 or more times a day

\section{How often did the following occur before COVID-19 affected your community?}

You read to [initial_child2] (e.g., you read aloud and your child

$\bigcirc$ listened)

You read with [initial child2] (e.g., child read the text with you) [initial child2] read independently (e.g., child read a book without help from you)

(2)
$\bigcirc$
$\bigcirc$
$\bigcirc$

$\bigcirc$

$\bigcirc$

$\bigcirc$

How often has the following
You read to [initial child2] (e.g.,
you read aloud and your child

listens)

You read with [initial_child2]

(e.g., child reads the text with you)

\section{[initial_child2] reads}

independently (e.g., child reads

a book without help from you)

\section{occurred since COVID-19 affected your community?}

\section{Never}

1 - 3 times

per month

1 - 2 times
per week

1 - 2 times
per week

$\bigcirc$

$\bigcirc$

$\bigcirc$

O

- 4 times
per week

Almost every day
1 - 2 times 3 or more per day times a day
On a typical day, how much was [initial_child2] read to before COVID-19 affected your community?
$1-30 \mathrm{~min}$

$31-60 \mathrm{~min}(1 \mathrm{hr})$

$61-90 \min (1.5 \mathrm{hrs})$

91 - $120 \mathrm{~min}$ (2 hrs)

$121-150 \mathrm{~min}(2.5 \mathrm{hrs})$

151 - 180 min (3 hrs)

Over 3 hrs

Not applicable 
On a typical day, how much is [initial_child2] read to since COVID-19 affected your community?
$1-30 \mathrm{~min}$
$31-60 \mathrm{~min}(1 \mathrm{hr})$
61 - $90 \mathrm{~min}$ (1.5 hrs)
91 - 120 min (2 hrs)
$121-150 \mathrm{~min}(2.5 \mathrm{hrs})$
151 - $180 \mathrm{~min}$ (3 hrs)
Over 3 hrs
Not applicable

At what age was [initial_child3] first read to?
Prenatal
0 - 4 months
5 - 9 months
10 - 14 months
15 - 19 months
20 - 24 months
Older than 24 months
Unknown/unsure

How often was [initial_child3] read to before COVID-19 affected your community?
Never
1 - 3 times per month
1 - 2 times per week
3 - 4 times per week
Almost every day
1 - 2 times per day
3 or more times a day

How often is [initial child3] read to since COVID-19

affected your community?
Never

1 - 3 times per month

1 - 2 times per week

3 - 4 times per week

Almost every day

1 - 2 times per day

3 or more times a day

\section{How often did the following occur before COVID-19 affected your community?}

\begin{tabular}{|c|c|c|c|c|c|c|}
\hline Never & $\begin{array}{l}1 \text { - } 3 \text { times } \\
\text { per month }\end{array}$ & $\begin{array}{l}1 \text { - } 2 \text { times } \\
\text { per week }\end{array}$ & $\begin{array}{l}\text { 3- } 4 \text { times } \\
\text { per week }\end{array}$ & $\begin{array}{l}\text { Almost } \\
\text { every day }\end{array}$ & $\begin{array}{c}1 \text { - } 2 \text { times } \\
\text { per day }\end{array}$ & $\begin{array}{l}3 \text { or more } \\
\text { times a day }\end{array}$ \\
\hline
\end{tabular}

You read to [initial_child3] (e.g., you read aloud and your child listened)

You read with [initial_child3] (e.g., child read the text with you)

[initial_child3] read

independently (e.g., child read a

book without help from you)
○

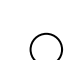

$\bigcirc$
$\bigcirc$

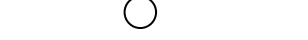

\section{O}

per

$\bigcirc$

$\bigcirc$

$\bigcirc$

.

$\begin{array}{ll}0 & 0 \\ 0 & 0\end{array}$




\section{How often has the following occurred since COVID-19 affected your community?}

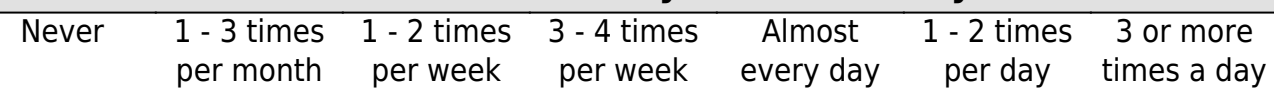

You read to [initial_child3] (e.g., you read aloud and your child

$\mathrm{O}$

$\bigcirc$

$\bigcirc$

listens)

You read with [initial_child3] (e.g., child reads the text with you)

[initial_child3] reads independently (e.g., child reads a book without help from you)

On a typical day, how much was [initial_child3] read to before COVID-19 affected your community?
1- $30 \mathrm{~min}$
$31-60 \min (1 \mathrm{hr})$
$\bigcirc 61-90 \mathrm{~min}$ (1.5 hrs)
$\bigcirc 91-120 \mathrm{~min}$ (2 hrs)
121 - $150 \mathrm{~min}$ ( $2.5 \mathrm{hrs}$ )
151 - $180 \mathrm{~min}$ (3 hrs)
$\bigcirc$ Over 3 hrs
$\bigcirc$ Not applicable

On a typical day, how much is [initial_child3] read to since COVID-19 affected your community?
$1-30 \mathrm{~min}$
$31-60 \mathrm{~min}(1 \mathrm{hr})$
$\bigcirc 61$ - $90 \mathrm{~min}$ (1.5 hrs)
91 - 120 min (2 hrs)
$\bigcirc 121-150 \mathrm{~min}$ (2.5 hrs)
O 151 - $180 \mathrm{~min}$ ( $3 \mathrm{hrs}$ )
$\bigcirc$ Over 3 hrs
Not applicable

At what age was [initial_child4] first read to?
Prenatal
0 - 4 months
5 - 9 months
10 - 14 months
15 - 19 months
20 - 24 months
Older than 24 months
Unknown/unsure

How often was [initial_child4] read to before COVID-19 affected your community?
Never
1 - 3 times per month
1 - 2 times per week
3 - 4 times per week
Almost every day
1 - 2 times per day
3 or more times a day 
How often is [initial_child4] read to since COVID-19 affected your community?

$\bigcirc$ Never

1 - 3 times per month

1 - 2 times per week

3 - 4 times per week

Almost every day

1 - 2 times per day

3 or more times a day

\section{How often did the following occur before COVID-19 affected your community?}

You read to [initial_child4] (e.g., you read aloud and your child listened)

You read with [initial_child4] (e.g., child read the text with you) [initial child4] read independently (e.g., child read a book without help from you)
O<smiles>O</smiles>

$\bigcirc$

$\bigcirc$ O

0

$\bigcirc$

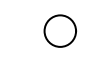<smiles>O</smiles><smiles>O</smiles>

O

O

d a

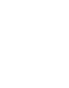

\section{How often has the following}

\section{Never}

\section{1 - 3 times \\ per month}

\section{VID-19 affected you
1 - 2 times 3 - 4 times \\ per week \\ per week}

\section{community?}

Almost 1 - 2 times 3 or more

per day
You read to [initial_child4] (e.g., you read aloud and your child listens)

You read with [initial_child4] (e.g., child reads the text with you)

[initial_child4] reads independently (e.g., child reads a book without help from you)
O

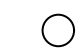

$\bigcirc$

$\bigcirc$

$\bigcirc$

$\bigcirc$
every day times a day

On a typical day, how much was [initial_child4] read to before COVID-19 affected your community?
$1-30 \mathrm{~min}$

$31-60 \mathrm{~min}(1 \mathrm{hr})$

$61-90 \mathrm{~min}$ (1.5 hrs)

$91-120 \mathrm{~min}$ (2 hrs)

$121-150 \mathrm{~min}$ (2.5 hrs)

151 - $180 \mathrm{~min}$ (3 hrs)

$\bigcirc$ Over 3 hrs

Not applicable 
On a typical day, how much is [initial_child4] read to since COVID-19 affected your community?
$1-30 \mathrm{~min}$
$31-60 \mathrm{~min}(1 \mathrm{hr})$
61 - $90 \mathrm{~min}$ (1.5 hrs)
91 - 120 min (2 hrs)
$121-150 \mathrm{~min}$ (2.5 hrs)
151 - $180 \mathrm{~min}$ (3 hrs)
Over 3 hrs
Not applicable

At what age was [initial_child5] first read to?
Prenatal
0 - 4 months
5 - 9 months
10 - 14 months
15 - 19 months
20 - 24 months
Older than 24 months
Unknown/unsure

How often was [initial_child5] read to before COVID-19 affected your community?
Never
1 - 3 times per month
1 - 2 times per week
3 - 4 times per week
Almost every day
1 - 2 times per day
3 or more times a day

How often is [initial_child5] read to since COVID-19

affected your community?
Never
1 - 3 times per month
1 - 2 times per week
3 - 4 times per week
Almost every day
1 - 2 times per day
3 or more times a day

\section{How often did the following occur before COVID-19 affected your community?

$\begin{array}{llccccc}\text { Never } & 1-3 \text { times } & 1-2 \text { times } & 3-4 \text { times } & \text { Almost } & 1-2 \text { times } & 3 \text { or more } \\ & \text { per month } & \text { per week } & \text { per week } & \text { every day } & \text { per day times a day }\end{array}$

You read to [initial_child5] (e.g., you read aloud and your child

$\bigcirc$ listened)

You read with [initial_child5] (e.g., child read the text with you)

[initial_child5] read

independently (e.g., child read a

book without help from you)

$\begin{array}{cccc}0 & 0 & 0 & 0 \\ 0 & 0 & 0 & 0 \\ 0 & 0 & 0 & 0\end{array}$




\section{How often has the following occurred since COVID-19 affected your community?}

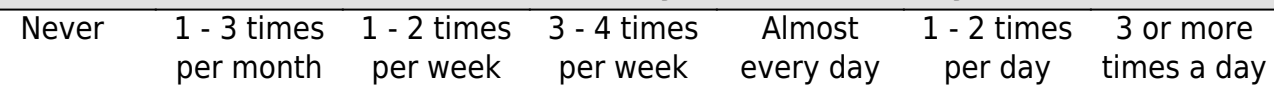

You read to [initial_child5] (e.g., you read aloud and your child

$\bigcirc$

O

listens)

You read with [initial_child5] (e.g., child reads the text with you)

[initial_child5] reads independently (e.g., child reads a book without help from you)

On a typical day, how much was [initial_child5] read to before COVID-19 affected your community?

1 - $30 \mathrm{~min}$

$31-60 \mathrm{~min}(1 \mathrm{hr})$

$61-90$ min (1.5 hrs)

91 - $120 \mathrm{~min}$ (2 hrs)

○ $121-150 \mathrm{~min}(2.5 \mathrm{hrs})$

151 - $180 \mathrm{~min}$ ( $3 \mathrm{hrs}$ )

Over 3 hrs

Not applicable

On a typical day, how much is [initial_child5] read to since COVID-19 affected your community?
$1-30 \mathrm{~min}$
$31-60 \mathrm{~min}(1 \mathrm{hr})$
61 - 90 min (1.5 hrs)
91 - $120 \mathrm{~min}$ (2 hrs)
$121-150 \mathrm{~min}$ ( $2.5 \mathrm{hrs})$
151 - $180 \mathrm{~min}$ (3 hrs)
$\bigcirc$ Over 3 hrs
$\bigcirc$ Not applicable

Please review your responses for completion and accuracy. You cannot go back once you've hit "Submit".

Survey completion: 3/6 


\section{Home Literacy Resources}




\section{Home Literacy Resources}

left: example of 25 children's books | right: example of 50 children's books

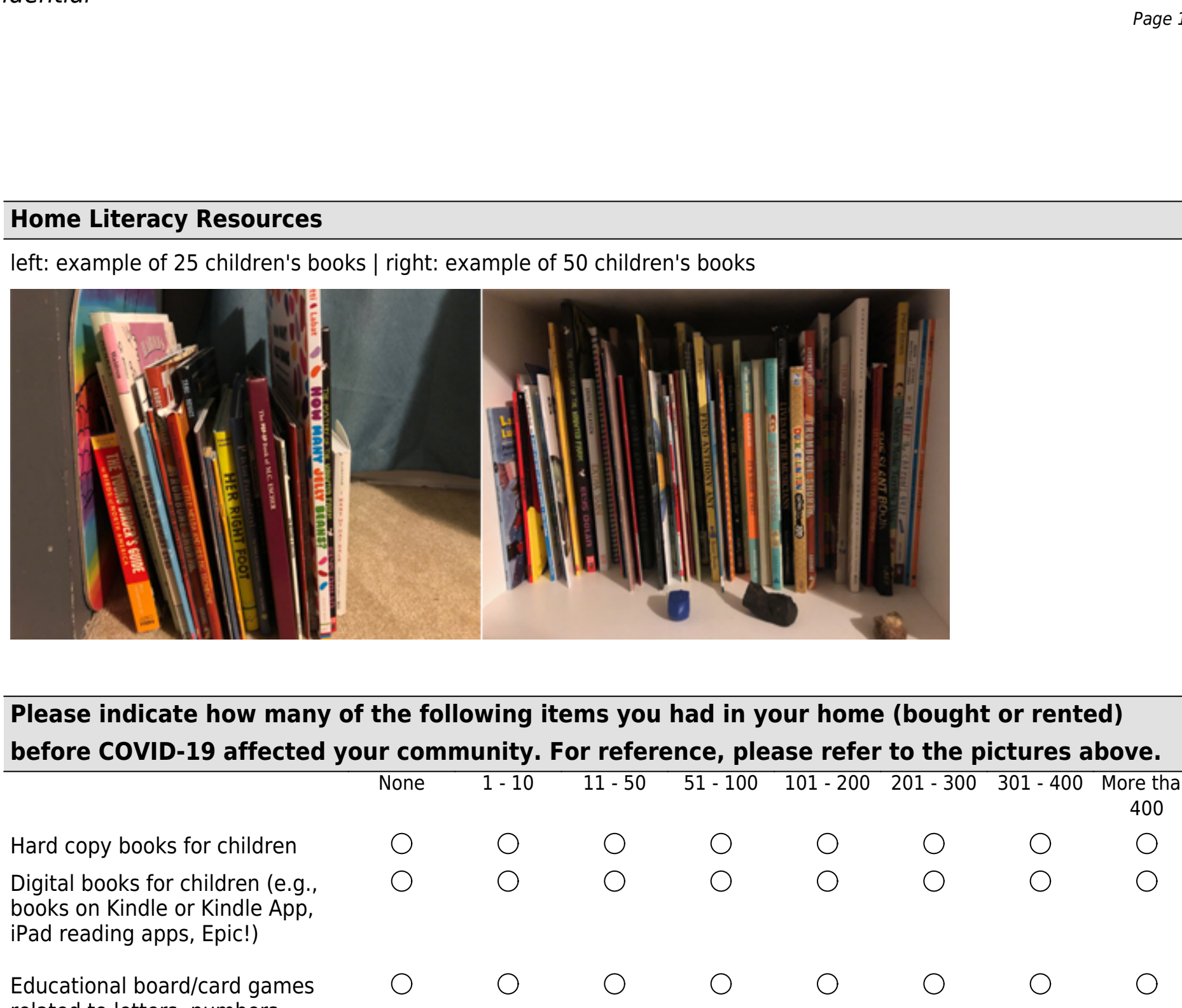

Please indicate how many of the following items you had in your home (bought or rented) before COVID-19 affected your community. For reference, please refer to the pictures above. $\begin{array}{llllllll}\text { None } & 1-10 & 11-50 & 51-100 & 101-200 & 201-300 & 301-400 & \text { More than }\end{array}$ 400

Hard copy books for children Digital books for children (e.g., books on Kindle or Kindle App, iPad reading apps, Epic!)

Educational board/card games related to letters, numbers, reading or math for children (e.g., Scrabble Jr., Boggle, Cranium, Educational Insights Games)

Digital educational games related to letters, numbers, reading or math for children (e.g., Hungry Caterpillar Play School, Elmo Loves 123s, PBS Kids Games)

Hard copy books for adults Digital books or newspaper subscriptions for adults (e.g., books on Kindle or Kindle App, iPad reading apps)

0

$\begin{array}{ll}0 & 0 \\ 0 & \bigcirc\end{array}$

$\bigcirc$
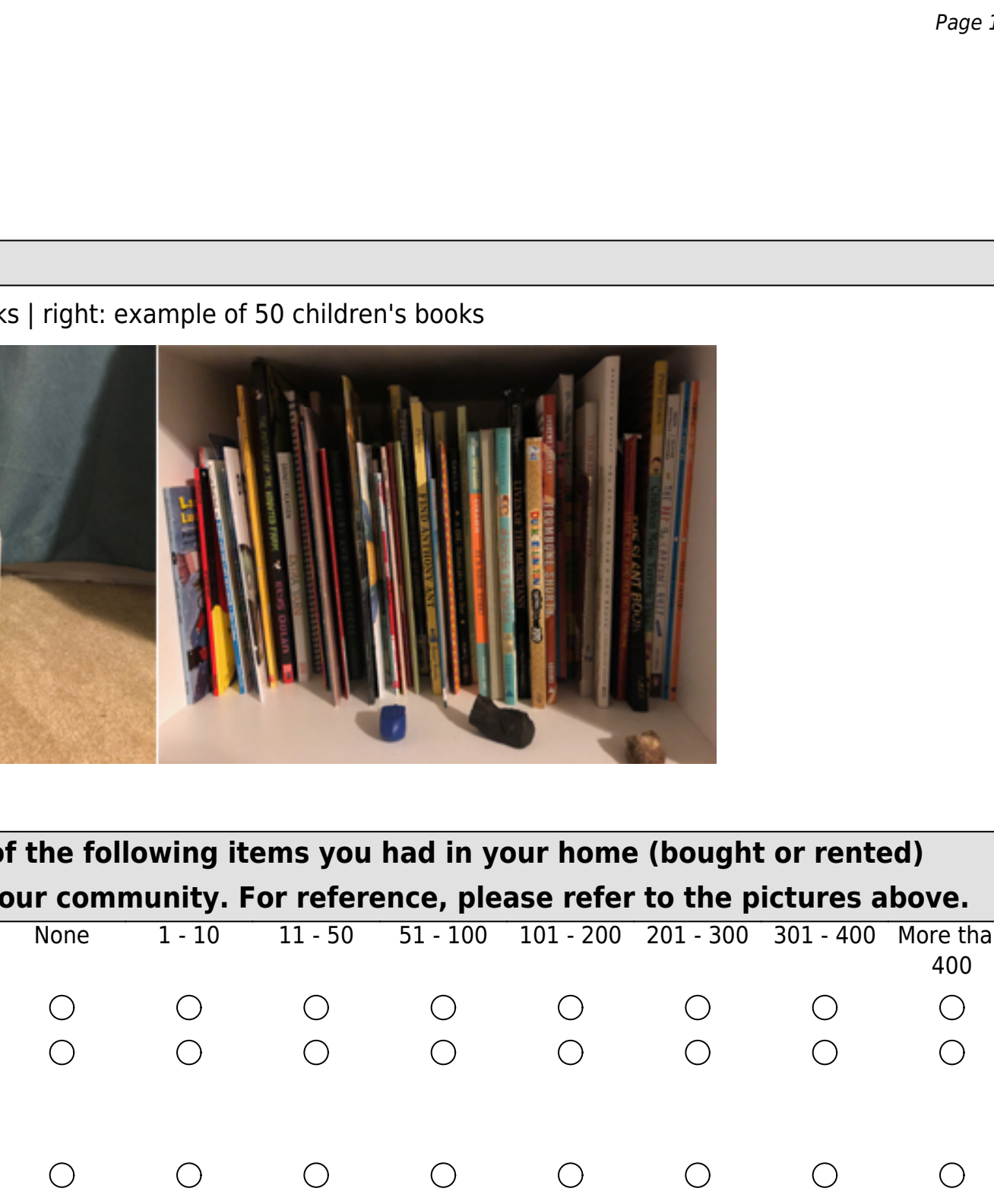
0

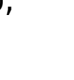

$\bigcirc$

s


Newspaper subscriptions for adults (digital or hard copy; e.g., The New York Times, The Boston Globe)

\section{Please indicate how many of the following items you have in your home (bought or rented) since COVID-19 affected your community. For reference, please refer to the pictures above.} $\begin{array}{llllllll}\text { None } & 1-10 & 11-50 & 51-100 & 101-200 & 201-300 & 301-400 & \text { More than }\end{array}$

Hard copy books for children

Digital books for children (e.g., books on Kindle or Kindle App, iPad reading apps, Epic!)

Educational board/card games related to letters, numbers, reading or math for children (e.g., Scrabble Jr., Boggle, Cranium, Educational Insights Games)

Digital educational games related to letters, numbers, reading or math for children (e.g., Hungry Caterpillar Play School, Elmo Loves 123s, PBS Kids Games)

Hard copy books for adults Digital books for adults (e.g., books on Kindle or Kindle App, iPad reading apps)

Newspaper subscriptions for adults (digital or hard copy; e.g., The New York Times, The Boston Globe)

$\begin{array}{ll}\bigcirc & 0 \\ 0 & 0\end{array}$

O

0

0

$\bigcirc \quad \bigcirc$

(n)




\section{Approximately how many of the following items did you accumulate since COVID-19 affected} your community?

Hard copy books for children:

Digital books for children (e.g., books on Kindle or Kindle App, iPad reading apps, Epic!)

Educational board/card games related to letters, numbers, reading or math for children (e.g.,

Scrabble Jr., Boggle, Cranium, Educational Insights Games)

Digital educational games related to letters, numbers, reading or math for children (e.g., Hungry Caterpillar Play School, Elmo Loves 123s, PBS Kids Games)

Hard copy books for adults

Digital books for adults (e.g., books on Kindle or Kindle App, iPad reading apps)

Please review your responses for completion and accuracy. You cannot go back once you've hit "Submit".

Survey completion: $4 / 6$ 


\section{Enrichment Activities}


Enrichment Activities

How often did [initial_child1] engage in the following activities in the home before COVID-19 affected your community?

$\begin{array}{ccccccc}\text { Never } & 1-3 \text { times } & 1-2 \text { times } & 3-4 \text { times } & \text { Almost } & 1-2 \text { times } & 3 \text { or more } \\ & \text { per month } & \text { per week } & \text { per week } & \text { every day } & \text { per day } & \text { times per }\end{array}$

per month per week per week every day per day times

Asked someone to read to them (e.g., asks adult to read a story at night)

Read books independently, or pretended to (e.g., reading favorite book without help from adult)

Played with magnetic letters or letter toys/cards

Watched educational TV shows or online videos (e.g., Sesame Street, ABC Mouse)

Actively played educational board/card games (e.g.,

Scrabble Jr., Zingo, Educational Insights Games)

Actively played educational video or computer games (e.g., PBS Kids Games,

ABCmouse.com)

Actively played on educational apps on a tablet (e.g., Hungry Caterpillar Play School, Elmo Loves 123s, PBS Kids Games)

$0 \quad 0$

$\bigcirc$

.


Reads books independently, or pretends to (e.g., reading favorite book without help from adult)

Play with magnetic letters or letter toys/cards

Watch educational TV shows or online videos (e.g., Sesame

Street, ABC Mouse)

Actively play educational board/card games (e.g., Scrabble Jr., Zingo, Educational Insights Games)

Actively play educational video or computer games (e.g., PBS Kids Games, ABCmouse.com)

Actively play on educational apps on a tablet (e.g., Hungry Caterpillar Play School, Elmo Loves 123s, PBS Kids Games) 
How often did [initial_child1] engage in the following activities in the home before COVID-19 affected your community?

Asked someone to read to them (e.g., asks adult to read a story at night)

Read books independently, or pretended to (e.g., reading favorite book without help from adult)

Watched educational TV shows or online videos related to reading and math (e.g., Sesame Street, ABC Mouse)

Watched TV with only subtitles (no sound)

Actively played educational board/card games related to reading and math (e.g., Boggle, Apples to Apples Junior, Cranium)

Actively played educational video or computer games related to reading and math (e.g., online PBS Kids Games, Khan Academy, BrainPop)

Actively played on educational apps on a tablet related to reading and math (e.g., app-based PBS Kids Games, Sago Mini Apps, DragonBox, Khan Academy App) 


\section{How often does [initial_child1] engage in the following activities in the home since COVID-19 affected your community?

Asks someone to read to them (e.g., asks adult to read a story at night)

Reads books independently, or pretends to (e.g., reading favorite book without help from adult)

Watches educational TV shows or online videos related to reading and math (e.g., Sesame Street, ABC Mouse)

Watches TV with only subtitles (no sound)

Actively plays educational board/card games related to reading and math (e.g., Boggle, Apples to Apples Junior, Cranium)

Actively played educational video or computer games related to reading and math (e.g., online PBS Kids Games, Khan Academy, BrainPop)

Actively played on educational apps on a tablet related to reading and math (e.g., app-based PBS Kids Games, Sago Mini Apps, DragonBox, Khan Academy App) 
How often did [initial_child2] engage in the following activities in the home before COVID-19 affected your community?

Asked someone to read to them (e.g., asks adult to read a story at night)

Read books independently, or pretended to (e.g., reading favorite book without help from adult)

Played with magnetic letters or letter toys/cards

Watched educational TV shows or online videos (e.g., Sesame Street, ABC Mouse)

Actively played educational board/card games (e.g.,

Scrabble Jr., Zingo, Educational Insights Games)

Actively played educational video or computer games (e.g., PBS Kids Games,

ABCmouse.com)

Actively played on educational apps on a tablet (e.g., Hungry Caterpillar Play School, Elmo Loves 123s, PBS Kids Games) 


\section{How often does [initial_child2] engage in the following activities in the home since COVID-19 affected your community?

Asks someone to read to them (e.g., asks adult to read a story at night)

Reads books independently, or pretends to (e.g., reading favorite book without help from adult)

Play with magnetic letters or letter toys/cards

Watch educational TV shows or online videos (e.g., Sesame Street, ABC Mouse)

Actively play educational board/card games (e.g., Scrabble Jr., Zingo, Educational Insights Games)

Actively play educational video or computer games (e.g., PBS Kids Games, ABCmouse.com)

Actively play on educational apps on a tablet (e.g., Hungry Caterpillar Play School, Elmo Loves 123s, PBS Kids Games) 
How often did [initial_child2] engage in the following activities in the home before COVID-19 affected your community?

Asked someone to read to them (e.g., asks adult to read a story at night)

Read books independently, or pretended to (e.g., reading favorite book without help from adult)

Watched educational TV shows or online videos related to reading and math (e.g., Sesame Street, ABC Mouse)

Watched TV with only subtitles (no sound)

Actively played educational board/card games related to reading and math (e.g., Boggle, Apples to Apples Junior, Cranium)

Actively played educational video or computer games related to reading and math (e.g., online PBS Kids Games, Khan Academy, BrainPop)

Actively played on educational apps on a tablet related to reading and math (e.g., app-based PBS Kids Games, Sago Mini Apps, DragonBox, Khan Academy App) 


\section{How often does [initial_child2] engage in the following activities in the home since COVID-19 affected your community?

$\begin{array}{ccccccc}\text { Never } & 1-3 \text { times } & 1-2 \text { times } & 3-4 \text { times } & \text { Almost } & 1-2 \text { times } & 3 \text { or more } \\ & \text { per month } & \text { per week } & \text { per week } & \text { every day } & \text { per day } & \text { times a day }\end{array}$

Asks someone to read to them (e.g., asks adult to read a story at night)

Reads books independently, or pretends to (e.g., reading favorite book without help from adult)

Watches educational TV shows or online videos related to reading and math (e.g., Sesame Street, ABC Mouse)

Watches TV with only subtitles (no sound)

Actively plays educational board/card games related to reading and math (e.g., Boggle, Apples to Apples Junior, Cranium)

Actively played educational video or computer games related to reading and math (e.g., online PBS Kids Games, Khan Academy, BrainPop)

Actively played on educational apps on a tablet related to reading and math (e.g., app-based PBS Kids Games, Sago Mini Apps, DragonBox, Khan Academy App) 
How often did [initial_child3] engage in the following activities in the home before COVID-19 affected your community?

Asked someone to read to them (e.g., asks adult to read a story at night)

Read books independently, or pretended to (e.g., reading favorite book without help from adult)

Played with magnetic letters or letter toys/cards

Watched educational TV shows or online videos (e.g., Sesame Street, ABC Mouse)

Actively played educational board/card games (e.g.,

Scrabble Jr., Zingo, Educational Insights Games)

Actively played educational video or computer games (e.g., PBS Kids Games,

ABCmouse.com)

Actively played on educational apps on a tablet (e.g., Hungry Caterpillar Play School, Elmo Loves 123s, PBS Kids Games) 


\section{How often does [initial_child3] engage in the following activities in the home since COVID-19 affected your community?

Asks someone to read to them (e.g., asks adult to read a story at night)

Reads books independently, or pretends to (e.g., reading favorite book without help from adult)

Play with magnetic letters or letter toys/cards

Watch educational TV shows or online videos (e.g., Sesame Street, ABC Mouse)

Actively play educational board/card games (e.g., Scrabble Jr., Zingo, Educational Insights Games)

Actively play educational video or computer games (e.g., PBS Kids Games, ABCmouse.com)

Actively play on educational apps on a tablet (e.g., Hungry Caterpillar Play School, Elmo Loves 123s, PBS Kids Games) 
How often did [initial_child3] engage in the following activities in the home before COVID-19 affected your community?

Never 1 - 3 times per month 1 - 2 times 3 - 4 times per week Almost every day 1 - 2 times per day 3 or more times per day

Asked someone to read to them (e.g., asks adult to read a story at night)

Read books independently, or pretended to (e.g., reading favorite book without help from adult)

Watched educational TV shows or online videos related to reading and math (e.g., Sesame Street, ABC Mouse)

Watched TV with only subtitles (no sound)

Actively played educational board/card games related to reading and math (e.g., Boggle, Apples to Apples Junior, Cranium)

Actively played educational video or computer games related to reading and math (e.g., online PBS Kids Games, Khan Academy, BrainPop)

Actively played on educational apps on a tablet related to reading and math (e.g., app-based PBS Kids Games, Sago Mini Apps, DragonBox, Khan Academy App)

$\bigcirc \quad \bigcirc \quad \bigcirc$

O

$\bigcirc$

$\bigcirc$

$\bigcirc$

$\bigcirc$

$\bigcirc$

O

$\bigcirc$

O

(n)




\section{How often does [initial_child3] engage in the following activities in the home since COVID-19 affected your community?

Asks someone to read to them (e.g., asks adult to read a story at night)

Reads books independently, or pretends to (e.g., reading favorite book without help from adult)

Watches educational TV shows or online videos related to reading and math (e.g., Sesame Street, ABC Mouse)

Watches TV with only subtitles (no sound)

Actively plays educational board/card games related to reading and math (e.g., Boggle, Apples to Apples Junior, Cranium)

Actively played educational video or computer games related to reading and math (e.g., online PBS Kids Games, Khan Academy, BrainPop)

Actively played on educational apps on a tablet related to reading and math (e.g., app-based PBS Kids Games, Sago Mini Apps, DragonBox, Khan Academy App) 
How often did [initial_child4] engage in the following activities in the home before COVID-19 affected your community?

Never 1 - 3 times per month 1 - 2 times 3 - 4 times per week Almost every day 1 - 2 times 3 or more per week per day times per day

Asked someone to read to them (e.g., asks adult to read a story at night)

Read books independently, or pretended to (e.g., reading favorite book without help from adult)

Played with magnetic letters or letter toys/cards

Watched educational TV shows or online videos (e.g., Sesame Street, ABC Mouse)

Actively played educational board/card games (e.g.,

Scrabble Jr., Zingo, Educational Insights Games)

Actively played educational video or computer games (e.g., PBS Kids Games,

ABCmouse.com)

Actively played on educational apps on a tablet (e.g., Hungry Caterpillar Play School, Elmo Loves 123s, PBS Kids Games)

O

O

$\bigcirc$

O

$\bigcirc$

$\bigcirc$

O

$\bigcirc$

$\bigcirc$

$\bigcirc$

.

a

al




\section{How often does [initial_child4] engage in the following activities in the home since COVID-19 affected your community?

Asks someone to read to them (e.g., asks adult to read a story at night)

Reads books independently, or pretends to (e.g., reading favorite book without help from adult)

Play with magnetic letters or letter toys/cards

Watch educational TV shows or online videos (e.g., Sesame Street, ABC Mouse)

Actively play educational board/card games (e.g., Scrabble Jr., Zingo, Educational Insights Games)

Actively play educational video or computer games (e.g., PBS Kids Games, ABCmouse.com)

Actively play on educational apps on a tablet (e.g., Hungry Caterpillar Play School, Elmo Loves 123s, PBS Kids Games) 
How often did [initial_child4] engage in the following activities in the home before COVID-19 affected your community?

Asked someone to read to them (e.g., asks adult to read a story at night)

Read books independently, or pretended to (e.g., reading favorite book without help from adult)

Watched educational TV shows or online videos related to reading and math (e.g., Sesame Street, ABC Mouse)

Watched TV with only subtitles (no sound)

Actively played educational board/card games related to reading and math (e.g., Boggle, Apples to Apples Junior, Cranium)

Actively played educational video or computer games related to reading and math (e.g., online PBS Kids Games, Khan Academy, BrainPop)

Actively played on educational apps on a tablet related to reading and math (e.g., app-based PBS Kids Games, Sago Mini Apps, DragonBox, Khan Academy App) 


\section{How often does [initial_child4] engage in the following activities in the home since COVID-19 affected your community?

$\begin{array}{ccccccc}\text { Never } & 1-3 \text { times } & 1-2 \text { times } & 3-4 \text { times } & \text { Almost } & 1-2 \text { times } & 3 \text { or more } \\ & \text { per month } & \text { per week } & \text { per week } & \text { every day } & \text { per day } & \text { times a day }\end{array}$

Asks someone to read to them (e.g., asks adult to read a story at night)

Reads books independently, or pretends to (e.g., reading favorite book without help from adult)

Watches educational TV shows or online videos related to reading and math (e.g., Sesame Street, ABC Mouse)

Watches TV with only subtitles (no sound)

Actively plays educational board/card games related to reading and math (e.g., Boggle, Apples to Apples Junior, Cranium)

Actively played educational video or computer games related to reading and math (e.g., online PBS Kids Games, Khan Academy, BrainPop)

Actively played on educational apps on a tablet related to reading and math (e.g., app-based PBS Kids Games, Sago Mini Apps, DragonBox, Khan Academy App) 
How often did [initial_child5] engage in the following activities in the home before COVID-19 affected your community?

Asked someone to read to them (e.g., asks adult to read a story at night)

Read books independently, or pretended to (e.g., reading favorite book without help from adult)

Played with magnetic letters or letter toys/cards

Watched educational TV shows or online videos (e.g., Sesame Street, ABC Mouse)

Actively played educational board/card games (e.g.,

Scrabble Jr., Zingo, Educational Insights Games)

Actively played educational video or computer games (e.g., PBS Kids Games,

ABCmouse.com)

Actively played on educational apps on a tablet (e.g., Hungry Caterpillar Play School, Elmo Loves 123s, PBS Kids Games) 


\section{How often does [initial_child5] engage in the following activities in the home since COVID-19 affected your community?

Asks someone to read to them (e.g., asks adult to read a story at night)

Reads books independently, or pretends to (e.g., reading favorite book without help from adult)

Play with magnetic letters or letter toys/cards

Watch educational TV shows or online videos (e.g., Sesame Street, ABC Mouse)

Actively play educational board/card games (e.g., Scrabble Jr., Zingo, Educational Insights Games)

Actively play educational video or computer games (e.g., PBS Kids Games, ABCmouse.com)

Actively play on educational apps on a tablet (e.g., Hungry Caterpillar Play School, Elmo Loves 123s, PBS Kids Games) 
How often did [initial_child5] engage in the following activities in the home before COVID-19 affected your community?

Never 1 - 3 times per month 1 - 2 times 3 - 4 times per week Almost every day 1 - 2 times per day 3 or more times per

Asked someone to read to them (e.g., asks adult to read a story at night)

Read books independently, or pretended to (e.g., reading favorite book without help from adult)

Watched educational TV shows or online videos related to reading and math (e.g., Sesame Street, ABC Mouse)

Watched TV with only subtitles (no sound)

Actively played educational board/card games related to reading and math (e.g., Boggle, Apples to Apples Junior, Cranium)

Actively played educational video or computer games related to reading and math (e.g., online PBS Kids Games, Khan Academy, BrainPop)

Actively played on educational apps on a tablet related to reading and math (e.g., app-based PBS Kids Games, Sago Mini Apps, DragonBox, Khan Academy App) day

$\bigcirc \quad \bigcirc \quad \bigcirc$

$\bigcirc$

$\bigcirc$

$\bigcirc$

$\bigcirc$

$\bigcirc$

$\bigcirc$

O

$\bigcirc$

O

(n)




\section{How often does [initial_child5] engage in the following activities in the home since COVID-19 affected your community?

Asks someone to read to them (e.g., asks adult to read a story $\mathrm{O}$<smiles>[O]</smiles>
at night)

Reads books independently, or pretends to (e.g., reading favorite book without help from adult)

Watches educational TV shows or online videos related to reading and math (e.g., Sesame Street, ABC Mouse)

Watches TV with only subtitles (no sound)

Actively plays educational board/card games related to reading and math (e.g., Boggle, Apples to Apples Junior, Cranium)

Actively played educational video or computer games related to reading and math (e.g., online PBS Kids Games, Khan Academy, BrainPop)

Actively played on educational apps on a tablet related to reading and math (e.g., app-based PBS Kids Games, Sago Mini Apps, DragonBox, Khan Academy App)

\section{Please review your responses for completion and accuracy. You cannot go back once you've hit "Submit".}

Survey completion: 5/6 
Education, Music, Demographics 


\section{Educational and Musical Environments at Home}

Before COVID-19 affected your community, how often did adults in your household who provided daily care to your child(ren) also help them with their school work? $\bigcirc$ Never

1 - 2 times a week

3 - 4 times a week

O - 6 times a week

1 - 2 times a day

3 - 4 times a day

Over 5 times a day
Since COVID-19 affected your community, how often do adults in your household who provide daily care to your child(ren) also help them with their school work?
Never

1 - 2 times a week

O 3 - 4 times a week

O - 6 times a week

1 - 2 times a day

3 - 4 times a day

$\bigcirc$ Over 5 times a day

\section{Please answer the following questions about [initial_child1].}

Since COVID-19 affected your community, has your child received school work or lesson plans/assignments from school?
Yes

No
How many hours a day does your child spend doing school work?
Less than $1 \mathrm{hr}$

$1 \mathrm{hr}-1 \mathrm{hr} 59 \mathrm{~min}$

2 hrs - 2 hrs 59 min

$3 \mathrm{hrs}-3 \mathrm{hrs} 59 \mathrm{~min}$

$4 \mathrm{hrs}-4 \mathrm{hrs} 59 \mathrm{~min}$

$5 \mathrm{hrs}-5 \mathrm{hrs} 59 \mathrm{~min}$

O 6 hrs -6 hrs 59 min

More than $7 \mathrm{hrs}$
How often do you help your child complete these assignments (e.g., helping with homework, teaching a lesson)?
Never
1 - 2 times a week
O 3 - 4 times a week
5 - 6 times a week
1 - 2 times a day
3 - 4 times a day
$\bigcirc$ Over 5 times a day

Since COVID-19 affected your community, has your child received live formal instruction from his/her teachers and/or tutors (e.g., Zoom, Google Meet,

OYes

No Skype, etc.)?

In a typical week, for how long is your child receiving live formal instruction?
$1-30 \mathrm{~min}$
$31-60 \mathrm{~min}(1 \mathrm{hr})$
61 - 90 min (1.5 hrs)
91 - 120 min (2 hrs)
121 - $150 \mathrm{~min}$ (2.5 hrs)
151 - 180 min (3 hrs)
Over 3 hrs
Not applicable 


\section{Please answer the following questions about [initial_child2].}

Since COVID-19 affected your community, has your child received school work or lesson

Y Yes

plans/assignments from school?

No

How many hours a day does your child spend doing school work?
$\bigcirc$ Less than $1 \mathrm{hr}$
$1 \mathrm{hr}-1 \mathrm{hr} 59 \mathrm{~min}$
2 hrs - 2 hrs 59 min
$3 \mathrm{hrs}-3 \mathrm{hrs} 59 \mathrm{~min}$
4 hrs - 4 hrs 59 min
5 hrs -5 hrs 59 min
$6 \mathrm{hrs}-6 \mathrm{hrs} 59 \mathrm{~min}$
More than $7 \mathrm{hrs}$

How often do you help your child complete these assignments (e.g., helping with homework, teaching a lesson)?
Never

1 - 2 times a week

3 - 4 times a week

5 - 6 times a week

1 - 2 times a day

3 - 4 times a day

Over 5 times a day
Since COVID-19 affected your community, has your child received live formal instruction from his/her teachers and/or tutors (e.g., Zoom, Google Meet,

$\bigcirc$ Yes
No Skype, etc.)?
In a typical week, for how long is your child receiving live formal instruction?
$1-30 \mathrm{~min}$
$31-60 \mathrm{~min}(1 \mathrm{hr})$
61 - $90 \mathrm{~min}$ (1.5 hrs)
91 - $120 \mathrm{~min}$ (2 hrs)
O $121-150 \mathrm{~min}$ ( $2.5 \mathrm{hrs})$
151 - $180 \mathrm{~min}$ (3 hrs)
Over 3 hrs
Not applicable

\section{Please answer the following questions about [initial_child3].}

$\begin{array}{ll}\begin{array}{l}\text { Since COVID-19 affected your community, has your } \\ \text { child received school work or lesson }\end{array} & \bigcirc \text { Yes } \\ \text { plo }\end{array}$
plans/assignments from school?

How many hours a day does your child spend doing school work?
Less than $1 \mathrm{hr}$

$1 \mathrm{hr}-1 \mathrm{hr} 59 \mathrm{~min}$

2 hrs - 2 hrs 59 min

$3 \mathrm{hrs}-3 \mathrm{hrs} 59 \mathrm{~min}$

$4 \mathrm{hrs}-4 \mathrm{hrs} 59 \mathrm{~min}$

$5 \mathrm{hrs}-5 \mathrm{hrs} 59 \mathrm{~min}$

$6 \mathrm{hrs}-6 \mathrm{hrs} 59 \mathrm{~min}$

More than $7 \mathrm{hrs}$
How often do you help your child complete these assignments (e.g., helping with homework, teaching a (esson)?
Never

1 - 2 times a week

3 - 4 times a week

5 - 6 times a week

1 - 2 times a day

3 - 4 times a day

Over 5 times a day 
Since COVID-19 affected your community, has your child received live formal instruction from his/her teachers and/or tutors (e.g., Zoom, Google Meet, Skype, etc.)?

In a typical week, for how long is your child receiving live formal instruction?
1 - $30 \mathrm{~min}$
$31-60 \mathrm{~min}(1 \mathrm{hr})$
61 - 90 min (1.5 hrs)
$91-120 \min (2 \mathrm{hrs})$
O $121-150 \mathrm{~min}$ ( $2.5 \mathrm{hrs}$ )
151 - $180 \mathrm{~min}$ ( $3 \mathrm{hrs}$ )
Over 3 hrs
$\bigcirc$ Not applicable

\section{Please answer the following questions about [initial_child4].}

Since COVID-19 affected your community, has your child received school work or lesson

$\bigcirc$ Yes plans/assignments from school?

How many hours a day does your child spend doing school work?
$\bigcirc$ Less than $1 \mathrm{hr}$
1 hr - 1 hr 59 min
$2 \mathrm{hrs}-2 \mathrm{hrs} 59 \mathrm{~min}$
$3 \mathrm{hrs}-3 \mathrm{hrs} 59 \mathrm{~min}$
O 4 hrs - 4 hrs 59 min
$5 \mathrm{hrs}-5 \mathrm{hrs} 59 \mathrm{~min}$
6 hrs -6 hrs 59 min
More than 7 hrs

How often do you help your child complete these assignments (e.g., helping with homework, teaching a lesson)?
Never

O 1 times a week

O - 4 times a week

5 - 6 times a week

1 - 2 times a day

3 - 4 times a day

Over 5 times a day

Since COVID-19 affected your community, has your child received live formal instruction from his/her teachers and/or tutors (e.g., Zoom, Google Meet, Skype, etc.)?

In a typical week, for how long is your child receiving live formal instruction?
1 - $30 \mathrm{~min}$
$31-60 \mathrm{~min}(1 \mathrm{hr})$
$\bigcirc 61-90 \mathrm{~min}$ (1.5 hrs)
$\bigcirc 91-120 \mathrm{~min}$ (2 hrs)
○ $121-150 \mathrm{~min}(2.5 \mathrm{hrs})$
151 - $180 \mathrm{~min}$ ( $3 \mathrm{hrs}$ )
$\bigcirc$ Over 3 hrs
$\bigcirc$ Not applicable 


\section{Please answer the following questions about [initial_child5].}

Since COVID-19 affected your community, has your child received school work or lesson

Y Yes

plans/assignments from school?

No

How many hours a day does your child spend doing school work?
$\bigcirc$ Less than $1 \mathrm{hr}$
$1 \mathrm{hr}-1 \mathrm{hr} 59 \mathrm{~min}$
2 hrs - 2 hrs 59 min
$3 \mathrm{hrs}-3 \mathrm{hrs} 59 \mathrm{~min}$
$4 \mathrm{hrs}-4$ hrs $59 \mathrm{~min}$
5 hrs -5 hrs 59 min
$6 \mathrm{hrs}-6 \mathrm{hrs} 59 \mathrm{~min}$
More than $7 \mathrm{hrs}$

How often do you help your child complete these assignments (e.g., helping with homework, teaching a lesson)?
Never

1 - 2 times a week

3 - 4 times a week

5 - 6 times a week

1 - 2 times a day

3 - 4 times a day

Over 5 times a day
Since COVID-19 affected your community, has your child received live formal instruction from his/her teachers and/or tutors (e.g., Zoom, Google Meet,

$\bigcirc$ Yes
No Skype, etc.)?
In a typical week, for how long is your child receiving live formal instruction?
$1-30 \mathrm{~min}$
$31-60 \mathrm{~min}(1 \mathrm{hr})$
$61-90 \mathrm{~min}$ (1.5 hrs)
91 - $120 \mathrm{~min}(2 \mathrm{hrs})$
121 - $150 \mathrm{~min}$ (2.5 hrs)
151 - $180 \mathrm{~min}$ (3 hrs)
Over 3 hrs
Not applicable

These next questions allow us to learn about your child(ren)'s musical exposure and engagement at home. Note that some of these questions may not be relevant to your child(ren)'s age(s). We do not expect you to have done all of these activities with your child(ren). Please answer, to your best ability, based on the timeframe cited in each question - either right before COVID-19 affected your community, or since COVID-19 affected your community.

\section{Please answer the following questions about [initial_child1].On a typical day, how often was [initial_child1] exposed to the following before COVID-19 affected your community?}

\begin{tabular}{|c|c|c|c|c|c|c|c|}
\hline $1-30 \mathrm{~min}$ & $\begin{array}{c}31-60 \\
\min (1 \mathrm{hr})\end{array}$ & $\begin{array}{c}61-90 \\
\min (1.5 \\
\text { hrs })\end{array}$ & $\begin{array}{c}91-120 \\
\min (2 \\
\text { hrs })\end{array}$ & $\begin{array}{c}121-150 \\
\min (2.5 \\
\mathrm{hrs})\end{array}$ & $\begin{array}{c}151-180 \\
\min (3 \\
\text { hrs })\end{array}$ & Over 3 hrs & $\begin{array}{c}\text { Not } \\
\text { applicable }\end{array}$ \\
\hline
\end{tabular}

Recorded music (e.g., radio, CDs, MP3s)

$\bigcirc \quad \bigcirc$


Music on TV (e.g., YouTube music videos, children's TV that features music)

\section{On a typical day, how often is [initial_child1] exposed to the following since COVID-19 affected} your community?

\begin{tabular}{|c|c|c|c|c|c|c|c|}
\hline $1-30 \mathrm{~min}$ & $\begin{array}{c}31-60 \\
\min (1 \mathrm{hr})\end{array}$ & $\begin{array}{c}61-90 \\
\min (1.5 \\
\text { hrs })\end{array}$ & $\begin{array}{c}91-120 \\
\min (2 \\
\mathrm{hrs})\end{array}$ & $\begin{array}{c}121-150 \\
\min (2.5 \\
\text { hrs })\end{array}$ & $\begin{array}{c}151-180 \\
\min (3 \\
\text { hrs) }\end{array}$ & Over 3 hrs & $\begin{array}{c}\text { Not } \\
\text { applicable }\end{array}$ \\
\hline
\end{tabular}

Recorded music (e.g., radio, CDs, MP3s)

Music on TV (e.g., YouTube music videos, children's TV that features music)

On a typical day, how often did you sing to [initial_child1] before COVID-19 affected your community (you are singing and your child is not)?
$1-30 \mathrm{~min}$

$31-60 \mathrm{~min}(1 \mathrm{hr})$

61 - 90 min (1.5 hrs)

91 - 120 min (2 hrs)

$121-150 \mathrm{~min}(2.5 \mathrm{hrs})$

151 - $180 \mathrm{~min}$ (3 hrs)

Over 3 hrs

Not applicable
On a typical day, how often do you sing to [initial_child1] since COVID-19 affected your community (you are singing and your child is not)?
$1-30 \mathrm{~min}$

$31-60 \mathrm{~min}(1 \mathrm{hr})$

61 - $90 \mathrm{~min}$ (1.5 hrs)

$91-120 \mathrm{~min}(2 \mathrm{hrs})$

$121-150 \min (2.5 \mathrm{hrs})$

151 - $180 \min (3 \mathrm{hrs}$ )

Over 3 hrs

Not applicable

Thinking back to before social distancing measures were put in place, to what extent was [initial_child1] typically engaged in:

\section{Never}

Early childhood musical

experiences (such as

kindermusik, "mommy and me"

music classes)

Music events in the community

(such as children's concerts,

park concerts/festivals, etc.)
Weekly

Bimonthly

$\bigcirc$

$\bigcirc$

$\bigcirc$

(2)

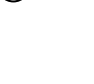

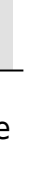


Music lessons (as may be relevant based on your child's age)

$\begin{aligned} & \text { Since social distancing measures were put in place, to what extent has [initial_child1] typically } \\ & \text { been engaged in: }\end{aligned}$
$\begin{array}{lllll}\text { Never } & \text { Weekly } & \text { Bimonthly } & \text { Monthly } & \begin{array}{c}\text { Several times a } \\ \text { year }\end{array}\end{array}$

Early childhood musical

experiences (such as

kindermusik, "mommy and me"

music classes)

Music events in the community

(such as children's concerts,

park concerts/festivals, etc.)

Music lessons (as may be

relevant based on your child's

age)

Please answer the following questions about [initial_child2].On a typical day, how often was [initial_child2] exposed to the following before COVID-19 affected your community?

$\begin{array}{cccccccc}1-30 \text { min } & 31-60 & 61-90 & 91-120 & 121-150 & 151-180 & \text { Over } 3 \text { hrs } & \text { Not } \\ & \min (1 \mathrm{hr}) & \min (1.5 & \min (2 & \min (2.5 & \min (3 & \text { applicable } \\ & & \mathrm{hrs}) & \mathrm{hrs}) & \mathrm{hrs}) & \mathrm{hrs}) & \end{array}$

Recorded music (e.g., radio,

CDs, MP3s)

Music on TV (e.g., YouTube

music videos, children's TV that

features music)

$\bigcirc \quad \bigcirc$

$\bigcirc$

$\bigcirc$

On a typical day, how often is [initial_child2] exposed to the following since COVID-19 affected your community?

$\begin{array}{cccccccc}1-30 \min & 31-60 & 61-90 & 91-120 & 121-150 & 151-180 & \text { Over 3 hrs } & \text { Not } \\ & \min (1 \mathrm{hr}) & \min (1.5 & \min (2 & \min (2.5 & \min (3 & \text { applicable } \\ & & \text { hrs }) & \text { hrs }) & \text { hrs }) & \text { hrs }) & \end{array}$

Recorded music (e.g., radio,

CDs, MP3s)

Music on TV (e.g., YouTube music videos, children's TV that

features music) 
On a typical day, how often did you sing to [initial_child2] before COVID-19 affected your community (you are singing and your child is not)?
$1-30 \mathrm{~min}$

$31-60 \min (1 \mathrm{hr})$

$61-90 \mathrm{~min}$ (1.5 hrs)

$91-120 \mathrm{~min}(2 \mathrm{hrs})$

121 - $150 \mathrm{~min}$ (2.5 hrs)

151 - 180 min (3 hrs)

Over 3 hrs

Not applicable
On a typical day, how often do you sing to [initial_child2] since COVID-19 affected your community (you are singing and your child is not)?
$1-30 \mathrm{~min}$
$31-60 \min (1 \mathrm{hr})$
61 - $90 \mathrm{~min}$ (1.5 hrs)
91 - $120 \mathrm{~min}(2 \mathrm{hrs})$
$121-150 \mathrm{~min}$ (2.5 hrs)
151 - $180 \mathrm{~min}$ (3 hrs)
Over 3 hrs
Not applicable

\section{Thinking back to before social distancing measures were put in place, to what extent was} [initial_child2] typically engaged in:

\section{Never}

Early childhood musical

experiences (such as

kindermusik, "mommy and me"

music classes)

Music events in the community

(such as children's concerts,

park concerts/festivals, etc.)

Music lessons (as may be relevant based on your child's age)

Weekly

Bimonthly

Monthly

Several times a

year

$\bigcirc$

$\bigcirc$

$\bigcirc$

$\bigcirc$

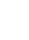


Please answer the following questions about [initial_child3].On a typical day, how often was

[initial_child3] exposed to the following before COVID-19 affected your community?

\begin{tabular}{|c|c|c|c|c|c|c|c|}
\hline $1-30 \mathrm{~min}$ & $\begin{array}{c}31-60 \\
\min (1 \mathrm{hr})\end{array}$ & $\begin{array}{c}61-90 \\
\min (1.5 \\
\text { hrs })\end{array}$ & $\begin{array}{c}91-120 \\
\min (2 \\
h r s)\end{array}$ & $\begin{array}{c}121-150 \\
\min (2.5 \\
h r s)\end{array}$ & $\begin{array}{c}151-180 \\
\min (3 \\
\text { hrs })\end{array}$ & Over 3 hrs & $\begin{array}{c}\text { Not } \\
\text { applicable }\end{array}$ \\
\hline
\end{tabular}

Recorded music (e.g., radio,

CDs, MP3s)

Music on TV (e.g., YouTube music videos, children's TV that features music)

$\bigcirc \bigcirc$

$\bigcirc$

O

$\bigcirc$

$\bigcirc \quad 0$

On a typical day, how often is [initial_child3] exposed to the following since COVID-19 affected your community?

\begin{tabular}{|c|c|c|c|c|c|c|c|}
\hline $1-30 \mathrm{~min}$ & $\begin{array}{c}31-60 \\
\min (1 \mathrm{hr})\end{array}$ & $\begin{array}{c}61-90 \\
\min (1.5 \\
\text { hrs) }\end{array}$ & $\begin{array}{c}91-120 \\
\min (2 \\
\text { hrs) }\end{array}$ & $\begin{array}{c}121-150 \\
\min (2.5 \\
\text { hrs })\end{array}$ & $\begin{array}{c}151-180 \\
\min (3 \\
\text { hrs) }\end{array}$ & Over 3 hrs & $\begin{array}{c}\text { Not } \\
\text { applicable }\end{array}$ \\
\hline
\end{tabular}

Recorded music (e.g., radio, CDs, MP3s)

Music on TV (e.g., YouTube music videos, children's TV that features music)

On a typical day, how often did you sing to [initial_child3] before COVID-19 affected your community (you are singing and your child is not)?
$1-30 \mathrm{~min}$

$31-60 \mathrm{~min}(1 \mathrm{hr})$

61 - 90 min (1.5 hrs)

$91-120 \mathrm{~min}$ (2 hrs)

$121-150 \min (2.5 \mathrm{hrs})$

151 - $180 \mathrm{~min}$ (3 hrs)

Over 3 hrs

Not applicable
On a typical day, how often do you sing to [initial_child3] since COVID-19 affected your community (you are singing and your child is not)?
$1-30 \mathrm{~min}$
$31-60 \mathrm{~min}(1 \mathrm{hr})$
$61-90 \mathrm{~min}$ (1.5 hrs)
91 - 120 min (2 hrs)
121 - $150 \mathrm{~min}$ (2.5 hrs)
151 - 180 min (3 hrs)
Over 3 hrs
Not applicable 
Thinking back to before social distancing measures were put in place, to what extent was [initial_child3] typically engaged in:

Never

Weekly

Bimonthly

Monthly

Several times a

year

Early childhood musical

experiences (such as

kindermusik, "mommy and me"

music classes)

Music events in the community

(such as children's concerts,

park concerts/festivals, etc.)

Music lessons (as may be

relevant based on your child's

age)

Since social distancing measures were put in place, to what extent has [initial_child3] typically
been engaged in:
$\begin{array}{lllll}\text { Never } & \text { Weekly } & \text { Bimonthly } & \text { Monthly } & \begin{array}{c}\text { Several times a } \\ \text { year }\end{array}\end{array}$

Early childhood musical

experiences (such as

kindermusik, "mommy and me"

music classes)

Music events in the community

(such as children's concerts,

park concerts/festivals, etc.)

Music lessons (as may be

relevant based on your child's

age)

\begin{tabular}{|c|c|c|c|c|c|c|c|}
\hline \multicolumn{8}{|c|}{$\begin{array}{l}\text { Please answer the following questions about [initial_child4].On a typical day, how often was } \\
\text { [initial_child4] exposed to the following before COVID-19 affected your community? }\end{array}$} \\
\hline $1-30 \mathrm{~min}$ & $\begin{array}{c}31-60 \\
\min (1 \mathrm{hr})\end{array}$ & $\begin{array}{l}61-90 \\
\min (1.5 \\
\text { hrs })\end{array}$ & $\begin{array}{l}91-120 \\
\min (2 \\
h r s)\end{array}$ & $\begin{array}{l}121-150 \\
\min (2.5 \\
\mathrm{hrs})\end{array}$ & $\begin{array}{l}151-180 \\
\min (3 \\
\mathrm{hrs})\end{array}$ & & $\begin{array}{c}\text { Not } \\
\text { applicable }\end{array}$ \\
\hline
\end{tabular}

Recorded music (e.g., radio,

CDs, MP3s)

Music on TV (e.g., YouTube

music videos, children's TV that

features music) 
On a typical day, how often is [initial_child4] exposed to the following since COVID-19 affected your community?

$\begin{array}{cccccccc}1-30 \min & 31-60 & 61-90 & 91-120 & 121-150 & 151-180 & \text { Over 3 hrs } & \text { Not } \\ & \min (1 \mathrm{hr}) & \min (1.5 & \min (2 & \min (2.5 & \min (3 & & \text { applicable } \\ & & \text { hrs }) & \mathrm{hrs}) & \mathrm{hrs}) & \mathrm{hrs}) & & \end{array}$

Recorded music (e.g., radio,

CDs, MP3s)

Music on TV (e.g., YouTube music videos, children's TV that features music)

On a typical day, how often did you sing to [initial_child4] before COVID-19 affected your community (you are singing and your child is not)?

$1-30 \mathrm{~min}$

$31-60 \mathrm{~min}(1 \mathrm{hr})$

61 - 90 min (1.5 hrs)

$\bigcirc 91-120 \mathrm{~min}$ (2 hrs)

$121-150 \mathrm{~min}$ ( $2.5 \mathrm{hrs}$ )

151 - 180 min (3 hrs)

$\bigcirc$ Over $3 \mathrm{hrs}$

$\bigcirc$ Not applicable

On a typical day, how often do you sing to

[initial_child4] since COVID-19 affected your

$\bigcirc 1-30 \mathrm{~min}$

community (you are singing and your child is not)?

$31-60 \mathrm{~min}(1 \mathrm{hr})$

61 - 90 min (1.5 hrs)

O 91 - 120 min (2 hrs)

$\bigcirc 121$ - $150 \mathrm{~min}$ ( $2.5 \mathrm{hrs}$ )

151 - 180 min (3 hrs)

Over 3 hrs

Not applicable

\section{Thinking back to before social distancing measures were put in place, to what extent was} [initial_child4] typically engaged in:

$$
\text { Never }
$$

Bimonthly

Monthly

Several times a year

Early childhood musical experiences (such as kindermusik, "mommy and me" music classes)

Music events in the community (such as children's concerts, park concerts/festivals, etc.)

Music lessons (as may be relevant based on your child's age)

$\bigcirc$

$\bigcirc$

\section{$\bigcirc$}

$\bigcirc$

$\bigcirc$ 
On a typical day, how often do you sing to [initial_child5] since COVID-19 affected your community (you are singing and your child is not)?
$1-30 \min$

$31-60 \min (1 \mathrm{hr})$

$61-90 \mathrm{~min}$ (1.5 hrs)

91 - 120 min (2 hrs)

121 - $150 \mathrm{~min}$ (2.5 hrs)

151 - $180 \mathrm{~min}$ (3 hrs)

Over 3 hrs

Not applicable

\section{Thinking back to before social distancing measures were put in place, to what extent was} [initial_child5] typically engaged in:

\section{Never}

Early childhood musical experiences (such as

kindermusik, "mommy and me" music classes)

Music events in the community (such as children's concerts, park concerts/festivals, etc.)

Music lessons (as may be relevant based on your child's age)

Weekly

Bimonthly

Monthly

Several times a

year

$\bigcirc$

$\bigcirc$

$\bigcirc$

(n)


Please indicate the geographic region you currently live in.
North America - United States of America

$\bigcirc$ North America - Canada

North America - Mexico

$\bigcirc$ Central and South America, including Peru, Brazil, Argentina, Guatemala, and surrounding countries

Ireland, Scotland or Wales

Northern Europe including Sweden, Norway, Finland and surrounding countries

$\bigcirc$ Western Europe including France, Germany, the Netherlands and surrounding countries

$\bigcirc$ Eastern Europe, including Russia, Poland, Hungary and surrounding countries

$\bigcirc$ Southern Europe including Italy, Greece, Spain, Portugal and surrounding countries

$\bigcirc$ Middle East including Lebanon, Turkey and surrounding countries

$\bigcirc$ Eastern Asia including China, Japan, South Korea, North Korea, Taiwan and Hong Kong

South East Asia including Thailand, Malaysia, Indonesia, Singapore and surrounding countries

South Asia including India, Pakistan, Sri Lanka and surrounding countries

$\bigcirc$ Oceania including Australia, New Zealand, Polynesia, Micronesia, Melanesia, Tonga, Fiji, Papua New Guinea and surrounding countries

$\bigcirc$ Northern Africa including Egypt, Libya, Algeria and Mauritania

Western Africa including Nigeria, Niger and Mali

$\bigcirc$ Central Africa including Chad, Central African Republic, Democratic Republic of the Congo

$\bigcirc$ Eastern Africa including Sudan, South Sudan, Uganda, Tanzania and Madagascar

Southern Africa including Angola, Zambia, Malawi and Mozambique

$\bigcirc$ Other

I prefer not to answer

Please specify:

Please indicate which geographic region in the United States you currently live in.
Far West (CA, NV, OR, WA)

Great Lakes (IL, IN, MI, OH, WI)

Mid Atlantic (DC, DE, MD, NJ, NY, PA)

$\bigcirc$ New England (CT, MA, ME, NH, RI, VT)

Plains (IA, KS, MN, MO, ND, NE, SD)

Rocky Mountain (CO, ID, MT, UT, WY)

Southeast (AL, AR, FL, GA, KY, LA, MS, NC, SC, TN, VA, WV)

Southwest (AZ, NM, OK, TX)

$\bigcirc$ Non-contiguous (AK, HI)

$\bigcirc$ U.S. territories (American Samoa, Guam, Northern Mariana Islands, Puerto Rico, U.S. Virgin Islands)
Which of the following best describes the area in which you live?
Large city

$\bigcirc$ Suburb of a large city

Small city

Town or village

Rural area 
Please indicate your household's current annual income. Please include all sources of income, such as wages, social security, unemployment benefits, insurance compensation, help from relatives, etc.
Less than $\$ 24,999$
$\$ 25,000-\$ 49,999$
$\$ 50,000-\$ 74,999$
$\$ 75,000-\$ 99,999$
$\$ 100,000-\$ 124,999$
$\$ 125,000-\$ 149,999$
$\$ 150,000-\$ 174,999$
$\$ 175,000-\$ 199,999$
$\$ 200,000-\$ 224,999$
$\$ 225,000-\$ 249,999$
$\$ 250,000-\$ 274,999$
$\$ 275,000-\$ 299,999$
$\$ 300,000$ or more

Eighth grade or less

Some high school

High school diploma or equivalency (GED)

Apprenticeship

Some college

$\bigcirc$ Two-year college degree or Associate's degree (junior college or vocational school)

$\bigcirc$ Four-year college degree or Bachelor's degree

$\bigcirc$ Some school beyond college

Graduate or professional degree

$\bigcirc$ Unknown or unsure

Not applicable [bg_caregivers_name_since_pg2].
Please indicate the highest degree earned of

Eighth grade or less

Some high school

High school diploma or equivalency (GED)

$\bigcirc$ Apprenticeship

Some college

$\bigcirc$ Two-year college degree or Associate's degree (junior college or vocational school)

$\bigcirc$ Four-year college degree or Bachelor's degree

Some school beyond college

Graduate or professional degree

$\bigcirc$ Unknown or unsure

Not applicable

Please indicate the highest degree earned of [bg_caregivers_name_since_pg3].
Eighth grade or less

Some high school

High school diploma or equivalency (GED)

$\bigcirc$ Apprenticeship

Some college

$\bigcirc$ Two-year college degree or Associate's degree (junior college or vocational school)

Four-year college degree or Bachelor's degree

$\bigcirc$ Some school beyond college

Graduate or professional degree

$\bigcirc$ Unknown or unsure

Not applicable 
Please indicate the highest degree earned of [bg_caregivers_name_since_pg4].
Eighth grade or less

Some high school

High school diploma or equivalency (GED)

Apprenticeship

Some college

Two-year college degree or Associate's degree (junior college or vocational school)

Four-year college degree or Bachelor's degree

Some school beyond college

Graduate or professional degree

Unknown or unsure

Not applicable
Please indicate [initial_child1]'s race(s). You may check all that apply. $\square$ American Indian or Alaska Native

Asian

Black or African American

$\square$ Native Hawaiian or Other Pacific Islander

White

Other

I prefer not to answer

Please specify:

Please indicate [initial_child2]'s race(s). You may check all that apply.

American Indian or Alaska Native

Asian

Black or African American

Native Hawaiian or Other Pacific Islander

White

Other

I prefer not to answer

Please specify:

Please indicate [initial_child3]'s race(s). You may check all that apply.
American Indian or Alaska Native

$\square$ Asian

Black or African American

Native Hawaiian or Other Pacific Islander

White

Other

I prefer not to answer

Please specify:

Please indicate [initial_child4]'s race(s). You may check all that apply. $\square$ American Indian or Alaska Native

$\square$ Asian

Black or African American

$\square$ Native Hawaiian or Other Pacific Islander

White

Other

I prefer not to answer

Please specify: 
Please indicate [initial_child5]'s race(s). You may check all that apply.
American Indian or Alaska Native

Asian

Black or African American

Native Hawaiian or Other Pacific Islander

White

Other

I prefer not to answer

Please specify:

Are you of Hispanic or Latino descent? (e.g. Mexican, Mexican-American, Chicano, Puerto Rican, Cuban, South or Central American, or other Spanish culture or origin)
Yes

No

I don't know

I prefer not to answer

Is [initial_child1] of Hispanic or Latino descent? (e.g., Mexican, Mexican-American, Chicano, Puerto Rican, Cuban, South or Central American, or other Spanish culture or origin)
Yes
No
I don't know
I prefer not to answer

Is [initial child2] of Hispanic or Latino descent? (e.g., Mexican, Mexican-American, Chicano, Puerto Yes Rican, Cuban, South or Central American, or other No Spanish culture or origin)

I don't know

I prefer not to answer

Is [initial_child3] of Hispanic or Latino descent? (e.g., Mexican, Mexican-American, Chicano, Puerto Rican, Cuban, South or Central American, or other Spanish culture or origin)

Yes
No
I don't know
I prefer not to answer

Is [initial_child4] of Hispanic or Latino descent? (e.g., Mexican, Mexican-American, Chicano, Puerto Rican, Cuban, South or Central American, or other Spanish culture or origin)
Y Yes
No
I don't know
I prefer not to answer

Is [initial_child5] of Hispanic or Latino descent? (e.g., Mexican, Mexican-American, Chicano, Puerto Rican, Cuban, South or Central American, or other Spanish culture or origin)
$\bigcirc$ Yes
No
I don't know
I prefer not to answer

Please review your responses for completion and accuracy. You cannot go back once you've hit "Submit".

Survey completion: $6 / 6$ 